\title{
Stable characteristic evolution of generic 3-dimensional single-black-hole spacetimes
}

\author{
R. Gómez ${ }^{\mathrm{a}}$, L. Lehner ${ }^{\mathrm{a}}$, R. L. Marsa ${ }^{\mathrm{a}, \mathrm{b}}$, J. Winicour $^{\mathrm{a}}$ \\ A. M. Abrahams ${ }^{\text {, }}$, A. Anderson ${ }^{\mathrm{d}}$, P. Anninos ${ }^{\mathrm{c}}$, T. W. Baumgarte ${ }^{\mathrm{c}}$, N. T. Bishop ${ }^{\mathrm{e}}$, S. R. Brandt ${ }^{\mathrm{c}}$, J. C. Browne ${ }^{\mathrm{b}}$,

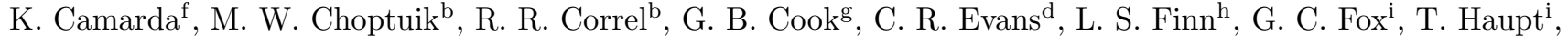 \\ M. F. Huq ${ }^{\text {, }}$ L. E. Kidder ${ }^{\text {h }}$, S. A. Klasky ${ }^{i}$, P. Laguna ${ }^{f}$, W. Landry ${ }^{\text {, }}$, J. Lenaghan ${ }^{\text {d }}$, J. Masso ${ }^{\text {c }}$, R. A. Matzner ${ }^{\text {, }}$, \\ S. Mitra ${ }^{b}$, P. Papadopoulos ${ }^{f}$, M. Parashar ${ }^{b}$, L. Rezzolla ${ }^{c}$, M. E. Rupright ${ }^{d}$, F. Saied ${ }^{\text {c }}$ P. E. Saylor ${ }^{\mathrm{c}}$, M. A. Scheel ${ }^{\mathrm{g}}$, \\ E. Seidel ${ }^{\text {, S. L. Shapiro }}{ }^{\text {, }}$ D. Shoemaker ${ }^{b}$, L. Smarr ${ }^{c}$, B. Szilágyia ${ }^{a}$, S. A. Teukolskyg , M. H. P. M. van Putten ${ }^{\text {, }}$, \\ P. Walker ${ }^{\mathrm{c}}$, J. W. York $\mathrm{Jr}^{\mathrm{d}}$. \\ ${ }^{a}$ University of Pittsburgh, Pittsburgh, Pennsylvania 15260 \\ ${ }^{\mathrm{b}}$ The University of Texas at Austin, Austin, Texas 78712 \\ ${ }^{\mathrm{c}}$ University of Illinois at Urbana-Champaign, Urbana, Illinois 61801 \\ ${ }^{\mathrm{d}}$ University of North Carolina, Chapel Hill, North Carolina 27599 \\ ${ }^{\mathrm{e}}$ University of South Africa, P.O. Box 392, Pretoria 0001, South Africa \\ ${ }^{\mathrm{f}}$ Penn State University, University Park, Pennsylvania 16802 \\ ${ }^{\mathrm{g}}$ Cornell University, Ithaca, New York 14853 \\ ${ }^{\mathrm{h}}$ Northwestern University, Evanston, Illinois 60208 \\ ${ }^{\mathrm{i}}$ Syracuse University, Syracuse, New York 13244-4100
}

We report new results which establish that the accurate 3dimensional numerical simulation of generic single-black-hole spacetimes has been achieved by characteristic evolution with unlimited long term stability. Our results cover a selection of distorted, moving and spinning single black holes, with evolution times up to $60,000 \mathrm{M}$.

04.25.Dm,04.30.Db

Accurate numerical simulation of black holes is necessary to calculate gravitational waveforms in the nonlinear regime that cannot be approximated by perturbation theory. The importance of such waveforms to the success of the LIGO gravity wave detector was a prime factor in organizing the Binary Black Hole Grand Challenge Alliance, whose goal is to provide the capability of obtaining waveforms from the inspiral and merger of binary black holes [1]. The Alliance is developing a code consisting of a Cauchy module matched to an outer boundary module using either a characteristic or perturbative method. For reports on the Cauchy and perturbative modules see [2] and [3], respectively. Here we report two new tests of a 3-dimensional characteristic evolution module [4 5] that establish its unlimited capability to accurately simulate a generic single black hole spacetime and that establish a calibrated tool to attack the binary problem: (i) We have evolved a black hole of mass $\mathrm{M}$ moving with periodic time dependence induced by a coordinate wobble for a time of $60,000 M$; and (ii) we have evolved an initially distorted, spinning black hole up to the final equilibrium state, which remains stationary to within machine roundoff error and is a discretized version of the Kerr black hole spacetime. (We use units with Newton's constant $G=1$ and the speed of light $c=1$. Thus $M \equiv G M / c^{3}$ is a time. Also, $M \equiv G M / c^{2}$ is a length.)

In the 1970s and 1980s, the difficulty of stably simulat- ing even a strictly spherical (one spatial dimension) single black hole led to the formulation of "the Holy Grail of numerical relativity", a list of requirements for " a code that simultaneously

- Avoids singularities

- Handles black holes

- Maintains high accuracy

- Runs forever." [6]

The results reported here definitely achieve this goal in the 3-dimensional, single black hole case. The challenge for the 1990s and beyond is the Binary Black Hole problem. The results here may become directly applicable to that multiple black hole stage.

The characteristic algorithm is a new computational treatment of hyperbolic systems. The theoretical framework is the characteristic initial value problem, pioneered by Bondi [7] and Penrose [8] in the 1960s. Almost all numerical modeling of hyperbolic systems has been based upon the Cauchy initial value problem, which evolves fields on spacelike hypersurfaces along a discrete sequence of time steps. The major new idea in the characteristic approach is to evolve fields on outgoing (or ingoing) light cones along a sequence of retarded (or advanced) time steps. Figure 1 shows the schematic setup for the outgoing case. A world tube $\Gamma$ has been placed as an inner boundary on the light cones (characteristics) to excise caustics from the evolution domain. Boundary data on $\Gamma$ and data on the initial light cone $\mathcal{N}_{0}$ determine a unique exterior evolution.

Characteristic evolution has several advantageous features [9]: The evolution variables reduce to one complex function related to the two gravitational polarization modes; the Einstein equations reduce to propagation equations along the light rays; and there are no constraints on the initial data. Furthermore, because the light cones are the spacetime hypersurfaces along which 
waves propagate, such propagating disturbances appear fairly smooth along them. This feature allows implementation of Penrose's spacetime compactification [8] to include points at future lightlike infinity (in the case based on outgoing light cones), where the waveform is calculated in the numerical grid. The major disadvantage is the difficulty in treating caustics. One early strategy for a characteristic algorithm proposed tackling the caustics head-on as part of the evolution [10]. But to date this has only been accomplished for point caustics in axisymmetric spacetimes [11]; and the extension to $3 \mathrm{D}$ would be prohibitive on present-day machines. Cauchy-characteristic matching is a strategy for combining the complementary strengths of Cauchy and characteristic evolution.

The implementation and calibration of the 3D characteristic module has been described elsewhere [4,12]. For a grid of discretization size $\Delta$, the numerical solutions converge in the continuum limit to exact analytic values in a wide variety of test beds, with $O\left(\Delta^{2}\right)$ error. The long term stability of the outgoing problem has also been established [4]. In these studies, the inner world tube $\Gamma$ was chosen to be the ingoing branch of the $r=2 M$ horizon in a Schwarzschild spacetime. The initial data consisted of a pulse of ingoing radiation on $\mathcal{N}_{0}$. This set the data for the scattering of a pulse of radiation by a Schwarzschild black hole, the classic problem first studied perturbatively by Price $\sqrt{13}$. The angular momentum of the ingoing pulse leads to a final black hole with spin. The pulse is partially transmitted into the black hole and partially scattered to (compactified) infinity along outgoing light cones, where its waveform is obtained. The evolution handles highly distorted black holes with backscattered radiation a thousand times more massive than the initial black hole and with a peak power $\approx 10^{5}$ in dimensionless units (equivalent to conversion of our galaxy's mass into gravitational waves in 1 second).

The calculation of the waveform at infinity for the binary problem can be posed in a similar way by matching a Cauchy interior module matched at a worldtube $\Gamma$ to a characteristic outer module. (See [3] for an alternative perturbative matching scheme). In model 3D nonlinear problems, Cauchy-characteristic matching dramatically outperforms other existing outer boundary conditions for Cauchy evolution $\sqrt{14}$. It has been successful in 1D general relativity [15, 16 but its efficacy in 3D general relativity is yet to be determined, because a stable matching scheme has not yet been found.

A simple transformation switches an outgoing characteristic evolution module into an ingoing module [5, 16. In this case, to uniquely define a black hole spacetime, boundary data is prescribed on an outer worldtube and on an incoming light cone ( $\Gamma$ and $\mathcal{N}_{0}$ in Fig. 2$)$; and in order to excise the singular region interior to the black hole, an inner boundary is constructed at a world tube traced out by a marginally trapped surface ( $\mathcal{T}$ in Fig. 2). This extends to characteristic evolution the strategy ini- tially proposed by Unruh (see [17) for Cauchy evolution of black holes.

This strategy is based upon the properties of trapped surfaces [18. Normally, the light rays emitted in the outward normal direction to a (topologically) spherical surface form an expanding beam. But strong gravitational lensing can make such an outgoing spherical beam everywhere convergent. Such a surface whose outgoing and ingoing rays all converge is called trapped. A marginally trapped surface (MTS) is the borderline case in which the outward light cone neither expands nor converges. Under reasonable assumptions, a MTS cannot lie outside a black hole (see [19). Consequently, if the worldtube $\Gamma$ in Fig. 2 is outside the black hole then the ingoing light cone $\mathcal{N}_{0}$ must extend some finite distance inward from $\Gamma$ before reaching a MTS ( $\mathcal{S}$ in Fig. 2). In all known examples of black holes the singularities are located inside a MTS. Excision of the interior of the MTS thus protects the evolution from encountering a singularity.

In order to implement this strategy (i) the evolution module must be equipped with an MTS finder and (ii) the singular region inside the MTS must be excised from the computational grid without influencing the exterior evolution. In a characteristic evolution, item (i) is facilitated by locating the MTS in a natural way by deforming an initial guess along the ingoing light rays [5]. Similarly, item (ii) is facilitated because the excision of the interior of the MTS reduces to a 1-dimensional problem with respect to a radial grid variable. There is no need for any further boundary condition on the MTS: by construction, waves emitted from its surface cannot expand into the exterior region. This theoretical property is built into the characteristic algorithm.

Details and calibration of the ingoing module are given in [5]. In initial simulations of a non-spinning black hole, data on $\Gamma$ was induced from the exterior geometry of a Schwarzschild spacetime and initial data on $\mathcal{N}_{0}$ consisted of a Schwarzschild black hole of mass $M$ distorted by a pulse of radiation. The worldtube $\Gamma$ was also placed in motion relative to the static symmetry of the exterior Schwarzschild spacetime to produce a time dependent location of the black hole in the numerical grid. The dynamics was monitored by tracking the surface area $\mathcal{A}$ of the MTS. For a non-spinning black hole in equilibrium, this surface area equals its Schwarzschild value $\mathcal{A}_{\mathcal{S}}=16 \pi M^{2}$. Calculation of $\mathcal{A}$ is an especially demanding test when the world tube is offset from the spherical symmetry of the Schwarzschild exterior and then placed in a periodic circular orbit. This periodic wobble of the coordinates leads to a periodic time dependence of both the metric and the location of the MTS, even in the final state of intrinsically static equilibrium. The area of a MTS determines its Hawking mass 20 and gives a useful measure of the energy inside it. Initially, $\mathcal{A}<\mathcal{A}_{\mathcal{S}}$ due to the energy content of the initial pulse on $\mathcal{N}_{0}$. The MTS grows as this energy falls into it. For a non-spinning 
black hole, $\mathcal{A} \rightarrow \mathcal{A}_{\mathcal{S}}$ as the MTS settles into equilibrium, even though the metric and the location of the MTS vary periodically (see Fig. 3).

It is important to establish that the ingoing characteristic module has no long term instabilities and that it can handle spinning black holes. We now present two new tests which demonstrate that it can essentially evolve a generic black hole "forever".

Since "forever" cannot be rigorously attained in any finite simulation, we appeal to a characteristic time necessary to obtain accurate waveforms for the inspiral and merger of two black holes. If one of the holes is small then the test particle approximation can be used. Consider a test particle in a quasi-circular orbit about a Schwarzschild black hole, where the final stable orbit is at $r=6 M$. From the quadrupole approximation (see [19]), the radiation rate per orbit is $\approx 10^{-2}$ of the binding energy, suggesting hundreds of orbits for the transition from inspiral to merger. The period measured by an observer at infinity is $12 \pi \sqrt{6} M \approx 90 M$ for this orbit so that the decay time would be $\approx 10,000 M$. For black holes of comparable mass, perturbation theory cannot reliably treat the regime intermediate between an orbital separation of $12 \mathrm{M}$ and merger. However, as estimated in 21] the decay time for this stage is $\approx 1500 M$ and to join the evolution smoothly to a post-Newtonian orbit at $20 M$ would require an evolution time of $\approx 10,000 \mathrm{M}$.

In our first test, we have successfully evolved an initially distorted, moving (but non-spinning) Schwarzschild black hole for a time of $60,000 M$, clearly as long as needed for a smooth transition from the postNewtonian regime to merger, if this success could be duplicated in the ultimate binary code. The run was terminated because it had achieved a steady state, with no sign of instability, and could be extended further. It was carried out in a wobbling coordinate system (see Fig. 3) which induces an "artificial" time dependence. (The wobble of the outer worldtube $\Gamma$ in the vicinity of $r=7 M$ is the only time dependence seen at late times in the evolution). This capability is important because it may not be possible to simulate a binary in coordinates which become exactly stationary after the merger and ring-down to final equilibrium.

Our second test establishes that the ingoing characteristic module handles spinning black holes. The outer world tube data is induced from the exterior geometry of a Kerr spacetime with mass $M$ and angular momentum parameter $a=M / 5$ (spin equal to $M^{2} / 5$ ). The metric is written in the Cartesian Kerr-Schild form [22]

$$
d s^{2}=-d t^{2}+d x^{2}+d y^{2}+d z^{2}+2 H k_{\mu} k_{\nu} d x^{\mu} d x^{\nu},
$$

where $k_{\mu}$ is tangent to an ingoing congruence of twisting light rays and $H$ is a potential (which equals $M / r$ in the $a=0$ nonspinning case). The outer worldtube $\Gamma$ is located at $x^{2}+y^{2}+z^{2}=49 M^{2}$. The module requires this worldtube data in Bondi coordinates, which are spherical coordinates based upon the light cones emanating inward from $\Gamma$ (advanced time coordinates). The transformation from Cartesian Kerr-Schild coordinates to spherical Bondi coordinates is carried out numerically in the neighborhood of the worldtube by an extraction module which forms part of the Alliance's Cauchy-characteristic matching procedure 12,23]. The initial data for a Kerr black hole on the ingoing light cone $\mathcal{N}_{0}$ is complicated to specify analytically (the geodesic equation leads to elliptic integrals). Instead, we choose initial data which approximates Kerr data but distorts the black hole. The amount of distortion can be measured in terms of the initial surface area of the MTS as compared with the Kerr value $\mathcal{A}_{K}=8 \pi M\left(M+\sqrt{M^{2}-a^{2}}\right)$. In Fig. 3, we plot $\mathcal{A}$ vs time for both the initially distorted Kerr and wobbling Schwarzschild cases. Tests show that at late times $\mathcal{A}$ converges to the exact (Kerr or Schwarzschild) value as the discretization size $\Delta \rightarrow 0$.

The Kerr evolution was run for a time of $15,000 M$, at which the only changes were at machine round-off. As apparent from Fig. 3, the final Kerr equilibrium is effectively reached at $20 \mathrm{M}$.

The success of the ingoing characteristic module suggests a possible strategy for excising the singularities in the binary case (see Fig. 4). Two disjoint characteristic evolutions based upon ingoing light cones are matched across worldtubes $\Gamma_{1}$ and $\Gamma_{2}$ to a Cauchy evolution of the shaded region between them. The ingoing light cones are each truncated at a MTS surrounding the singularities. The outer boundary $\Gamma$ of the Cauchy region is matched to an exterior characteristic evolution based upon outgoing light cones extending to infinity, where the waveform is calculated. This global strategy has been successfully implemented for spherically symmetric self-gravitating scalar waves evolving in a single black hole spacetime [16].

Just as several coordinate patches are necessary to describe a spacetime with nontrivial topology, an effective attack on the binary black hole problem could be to patch together regions of spacetime handled by different algorithms. The Cauchy-characteristic modules are in place and calibrated for accuracy. The Alliance's Cauchy module [2] has evolved a Schwarzschild black hole for a time of $475 \mathrm{M}$. Its performance and stability are now being studied in a wide variety of tests. (For Cauchy evolution of a black hole with another 3D code, see (24). The key missing ingredient is the long term stability of matching, which is a major current project.

This work was supported by the Binary Black Hole Grand Challenge Alliance, NSF PHY/ASC 9318152 (ARPA supplemented). Computer time was provided by the Pittsburgh Supercomputing Center. 


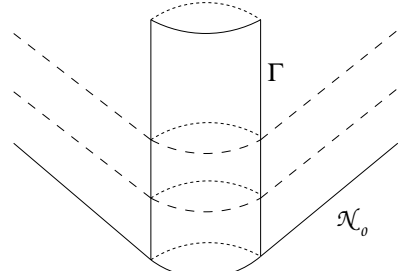

FIG. 1. The outgoing formulation: The exterior of $\Gamma$ is covered by a sequence of outgoing light cones.

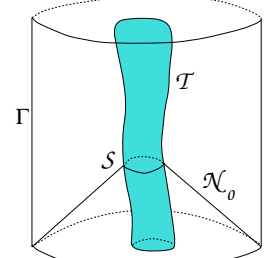

FIG. 2. The ingoing formulation: The interior of $\Gamma$ is covered by a sequence of ingoing light cones. The interior of $\mathcal{T}$ is excised from the evolution.

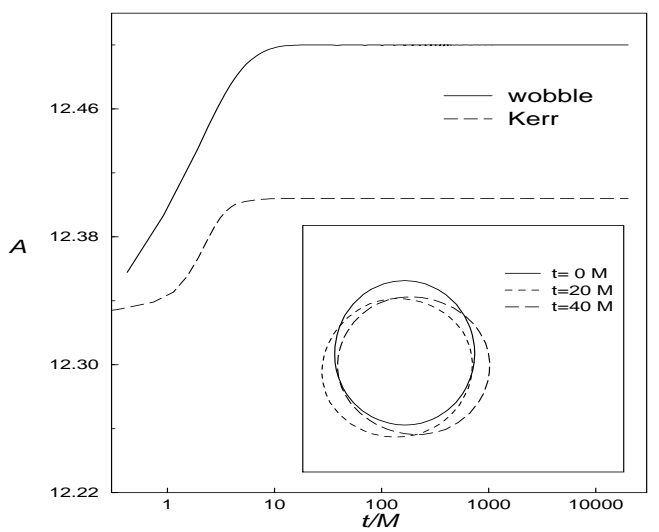

FIG. 3. Surface area $v s$ time for a wobbling hole (with rotation frequency 0.1 , offset 0.1 and mass 0.5 ) and an initially distorted spinning hole (Kerr mass 0.5). The inset shows three different snapshots of the MTS in the case of the "wobble".

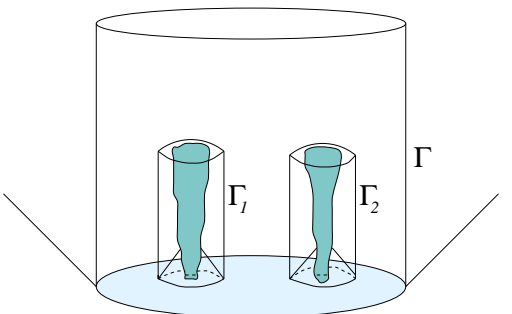

FIG. 4. A matching scheme for two orbiting black holes (in a co-rotating frame which eliminates the major source of time dependence).
[1] For information about the goals and status of the Alliance visit: http://www.npac.syr.edu/projects/bh/.

[2] The Binary Black Hole Grand Challenge Alliance, Boosted 3-dimensional black hole evolutions with singularity excision, (submitted for publication).

[3] The Binary Black Hole Grand Challenge Alliance, Schwarzschild-perturbative gravitational wave extraction and outer boundary conditions, Phys. Rev. Lett. (to appear).

[4] N.T. Bishop, R. Gómez, L. Lehner, M. Maharaj and J. Winicour, Phys. Rev. D 56, 6298 (1997).

[5] R. Gómez, L. Lehner, R. L. Marsa and J. Winicour, "Moving black holes in 3D", submitted for publication, gr-qc/9710138.

[6] S.L. Shapiro, S.A. Teukolsky, in Dynamical Spacetimes and Numerical Relativity, ed. J. Centrella (Cambridge UP, Cambridge, 1986) p. 74.

[7] H. Bondi, M.J.G. van der Burg and A.W.K. Metzner, Proc. R. Soc. Lond. A 269, 21, 1962.

[8] R. Penrose, Phys. Rev. Lett., 10, 66 (1963).

[9] R. Gómez and J. Winicour, in Approaches to Numerical Relativity, ed. R. d'Inverno (Cambridge University Press, Cambridge, 1992) p. 143.

[10] H. Friedrich and J.M. Stewart Proc. R. Soc. Lond. A, 385, 345 (1983).

[11] R. Gómez, P. Papadopoulos and J. Winicour, J. Math. Phys. 35, 4184, 1994.

[12] N.T. Bishop, R. Gómez, L. Lehner, and J. Winicour, Phys. Rev. D 54 6153, 1996.

[13] R.H. Price, Phys. Rev. D 5 2419, (1972).

[14] N.T. Bishop, R. Gómez, P.R. Holvorcem, R.A. Matzner, P. Papadopoulos, and J. Winicour, J. Comput. Phys. 136, 236 (1997).

[15] M. Dubal, R. d'Inverno and C. Clarke, Phys. Rev. D 52, 6868 (1995).

[16] R. Gómez, R. Marsa and J. Winicour, Phys. Rev. D 56, 6310 (1997).

[17] J. Thornburg, Class. Quantum Grav. 4, 1119 (1987).

[18] R. Penrose, Phys. Rev. Lett., 14, 57 (1965).

[19] R.M. Wald, General Relativity (University of Chicago Press, Chicago, 1984).

[20] S. W. Hawking, J. Math. Phys. 9, 598 (1968).

[21] É.É. Flanagan and S.W. Hughes, (submitted to Phys. Rev. D), gr-qc/9701039.

[22] R.P. Kerr and A. Schild, Proc. Symp. Appl. Math. 17199 (1965).

[23] N. T. Bishop, R. Gómez, R. A. Isaacson, L. Lehner, Bela Szilagyi and J. Winicour, "Cauchy Characteristic Matching", in On the Black Hole Trail, eds. B. Iyer and B. Bhawal (Kluwer, Dordrecht, to appear).

[24] P. Anninos, K. Carmada, J. Massó, E. Seidel, W.-M. Suen and J. Towns, Phys. Rev. D 52, 2059 (1995); P. Anninos, J. Massó, E. Seidel and W.-M. Suen, Physics World 9, 43 (1996). 